\title{
FPDHP, a novel anticancer agent, induces cell detachment and caspase-dependent apoptosis in Caki cells
}

\author{
JUN SOO PARK ${ }^{1 *}$, WAN TAE KIM ${ }^{1 *}$, SHIN KIM ${ }^{1}$, TAEG KYU KWON ${ }^{1}$, BYEONG-CHURL JANG ${ }^{1}$, \\ EUNG-SEOK LEE ${ }^{2}$ and JONG WOOK PARK ${ }^{1}$ \\ ${ }^{1}$ Chronic Disease Research Center, Keimyung University School of Medicine, Daegu 704-701; \\ ${ }^{2}$ College of Pharmacy, Yeungnam University, Kyongsan 712-749, Republic of Korea
}

Received March 31, 2014; Accepted July 16, 2014

DOI: $10.3892 /$ ijmm.2014.1875

\begin{abstract}
The inhibition of topoisomerase can suppress the growth of cancer cells and induce apoptosis. The aim of this study was to evaluate the anticancer effects and mechanisms of action of a novel topoisomerase inhibitor, 4-(furan-2-yl)-2-(pyridin2-yl)-5,6-dihydro-1,10-phenanthroline (FPDHP). FPDHP suppressed the growth of Caki, A549, HT29 and MDA-MB-231 cells, and induced caspase-dependent apoptosis in the Caki cells. In particular, FPDHP also induced caspase-dependent apoptosis and the downregulation of the protein expression levels of cellular FLICE-like inhibitory protein (cFLIP) and the phosphorylation of Akt in Caki cells. Notably, the overexpression of cFLIP, but not that of Akt, in part, blocked the FPDHP-mediated apoptosis in Caki cells. In addition, FPDHP was further shown to induce the caspase-independent detachment of Caki cells from the culture dish; higher populations of apoptotic cells were observed in the detached cells than in the attached cells. To the best of our knoweledge, these results collectively demonstrate for the first time that FPDHP has a killing effect on Caki cells, which is mediated through both caspase-dependent apoptosis and caspase-independent cell detachment.
\end{abstract}

\section{Introduction}

DNA topoisomerases are one of the most promising molecular targets for the development of anticancer agents (1). They are nuclear enzymes that transiently break one or two strands of DNA providing solutions to various DNA topological problems

Correspondence to: Professor Jong Wook Park, Chronic Disease Research Center, Keimyung University School of Medicine, 1095 Dalgubeol-daero, Dalseo-gu, Daegu 704-701, Republic of Korea E-mail: j303nih@dsmc.or.kr

Professor Eung-Seok Lee, College of Pharmacy, Yeungnam University, 214-1 Daedong, Kyongsan 712-749, Republic of Korea

E-mail: eslee@yu.ac.kr

*Contributed equally

Key words: caspase-dependent apoptosis, cell detachment 4-(furan-2-yl)-2-(pyridin-2-yl)-5,6-dihydro-1,10-phenanthroline associated with DNA replication, transcription, recombination and other vital cellular processes $(2,3)$. Due to the crucial role of topoisomerase in the maintenance and replication of DNA during proliferation, cells become highly vulnerable when these functions are lost (4). Therefore, topoisomerases are attractive targets for designing anticancer agents (5). DNA topoisomerases are generally classified into topoisomerases I and II, depending on their mechanisms of action, leading to either single or double-strand breaks, respectively (6).

Several synthetic compounds, such as benzoxanthone derivatives, thiosemicarbazones, benzophenanthridines, purine analogues, anilinothiazoloquinolines, benzofuroquinolinediones, coumarin derivatives and trisubstituted pyridines have been reported as topoisomerase inhibitors (7). Previously, our research group synthesized various rigid analogues of 2,4,6-trisubstituted pyridines and evaluated thse analogues for their topoisomerase inhibitory activity, as well as cytotoxicity, in order to determine the effects of rigid structure on anticancer activity (8-10). Rigid structures are commonly considered to have little conformational entropy compared to flexible structures, and can be more efficiently fitted into the active site of a receptor (11). It has been reported that planar molecules are able to intercalate into the DNA helix and stabilize the topoisomerase-DNA covalent cleavage, converting topoisomerase into a lethal DNA-damaging agent. Recently, we synthesized various phenanthroline derivatives possessing a quinoline core for the introduction of a rigid back bone bearing 5 membered aromatic rings, such as furyl or thienyl at the 4-position and evaluated these derivatives for their topoisomerase I and II inhibitory activity and cytotoxicity against several human cancer cell lines. Among the tested compounds, 4-(furan-2-yl)-2-(pyridin-2-yl)-5,6-dihydro-1,10-phenanthroline (FPDHP) was found to exhibit a significant and selective topoisomerase I inhibitory activity of $60.9 \%$ at a dose of $100 \mu \mathrm{M}$ which corresponded a 1.25-fold greater activity than camptothecin, and also to have strong cytotoxicity against several human cancer cells [DU145 (prostate), HCT115 (colon) and T47D (breast)] (12). However, the mechanisms underlying the FPDHP-mediated cytotoxicity against cancer cells remain elusive. In the present study, we investigated the inhibitory effects of FPDHP on the growth of various types of cancer cells, including Caki (kidney), A549 (lung), HT29 (colon) and MDA-MB-231 (breast) camcer cells, and determined the molecular and/or cellular mechanisms involved. 


\section{Materials and methods}

Cell lines and culture. The MDA-MB-231 and A549 cells (American Type Culture Collection, Manassas, VA, USA) were grown in RPMI-1640 medium supplemented with $10 \%$ heatedinactivated fetal bovine serum (FBS), $2 \mathrm{mM}$ L-glutamine, $100 \mu \mathrm{g} / \mathrm{ml}$ streptomycin and $100 \mu \mathrm{g} / \mathrm{ml}$ penicillin. The Caki (American Type Culture Collection) and HT29 cells (American Type Culture Collection) were grown in Dulbecco's modified Eagle's medium, containing 10\% heat-inactivated FBS, $20 \mathrm{mM}$ HEPES buffer and $100 \mu \mathrm{g} / \mathrm{ml}$ streptomycin and $100 \mu \mathrm{g} / \mathrm{ml}$ penicillin. Caki cells overexpressing cellular FLICE-like inhibitory protein (cFLIP) (Caki/cFLIP) or Akt (Caki/Akt) and the control cells (Caki/vector) were kindly supplied by Dr T.K. Kwon (Keimyung University, Daegu, Korea).

Drugs and materials. FPDHP was kindly supplied by Dr E.-S. Lee (Yeungnam University, Daegu, Korea) (Fig. 1A). The pan-caspase inhibitor, z-VAD-FMK (z-VAD), was purchased from Santa Cruz Biotechnology, Inc. (Santa Cruz, CA, USA). Anti-cFLIP, anti-B-cell lymphoma-2 (Bcl-2), antiphospholipase C (PLC)- $\gamma 1$ and anti-pro-caspase-3 antibodies were purchased from Santa Cruz Biotechnology, Inc. Anti-Akt and anti-phospho-Akt (p-Akt), anti-extracellular signal-related kinase (ERK) and anti-cleaved caspase-3 antibodies were purchased from Cell Signaling Technology (Beverly, MA, USA).

2,3-Bis(2-methoxy-4-nitro-5-sulfophenyl)-2H-tetrazolium-5carboxanilide inner salt (XTT) assay. Cell proliferation was detected by XTT assay (WelGENE Inc., Daegu, Korea). When the cultured cells were in the log phase, they were seeded on a 96 -well plate $\left(2 \times 10^{4}\right.$ cells/ $100 \mu \mathrm{l} /$ well) for $24 \mathrm{~h}$. The cells were then treated with or without FPDHP for $24 \mathrm{~h}$. Absorbance (A) was detected using an enzyme calibrator at $450 \mathrm{~nm}$. Relative cell growth $(\%)=($ A of study group $/ \mathrm{A}$ of control group $) \times 100$.

Western blot analysis. Caki cells seeded in a 6-well plate $\left(4 \times 10^{5}\right.$ cells $/ 4 \mathrm{ml} /$ well) the day before treatment were treated with or without FPDHP in the presence or absence of the pancaspase inhibitor, z-VAD, for the indicated periods of time. To prepare cellular lysates, the conditioned cells were initially exposed to a lysis buffer $(137 \mathrm{mM} \mathrm{NaCl}, 15 \mathrm{mMEGTA}, 0.1 \mathrm{mM}$ sodium orthovanadate, $15 \mathrm{mM} \mathrm{MgCl} 2,0.1 \%$ Triton X-100, $25 \mathrm{mM}$ MOPS, $100 \mathrm{mM}$ phenylmethylsulfonyl fluoride and $20 \mathrm{mM}$ leupeptin, adjusted to $\mathrm{pH}$ 7.2). The samples were further disrupted by sonication and extracted at $48^{\circ} \mathrm{C}$ for $30 \mathrm{~min}$. The lysates were centrifuged at $10,000 \times \mathrm{g}$ for $15 \mathrm{~min}$ at $48^{\circ} \mathrm{C}$, and the supernatant fractions were collected. Approximately $50 \mu \mathrm{g}$ of protein was separated by sodium dodecyl sulfatepolyacrylamide gel electrophoresis (SDS-PAGE), and electrotransferred onto Immobilon-P membranes (Millipore Corp., Billerica, MA, USA). The membranes were incubated with blocking buffer ( $0.1 \%$ Triton X-100 with 5\% non-fat dry milk in TBS) for 30 min. Following 3 washes with TBST, the membranes were incubated with primary antibody overnight. The membranes were washed 3 times with TBST, and incubated with HRP-conjugated secondary antibody. The detection of specific proteins was carried out using an ECL western blotting kit according to the manufacturer's instructions (Millipore Corp.).
Flow cytometric analysis. Approximately $1 \times 10^{6}$ Caki cells were suspended in $100 \mu \mathrm{l}$ phosphate-buffered saline (PBS), and $200 \mu \mathrm{l}$ of $95 \%$ ethanol were added while vortexing. The cells were incubated at $4^{\circ} \mathrm{C}$ for $1 \mathrm{~h}$, washed with PBS and resuspended in $250 \mu 1$ of $1.12 \%$ sodium citrate buffer ( $\mathrm{pH} 8.4$ ) together with $12.5 \mu \mathrm{g}$ RNase. Incubation was continued at $37^{\circ} \mathrm{C}$ for $30 \mathrm{~min}$. The cellular DNA was then stained by applying $250 \mu \mathrm{l}$ of propidium iodide $(50 \mu \mathrm{g} / \mathrm{ml})$ for $30 \mathrm{~min}$ at room temperature. The stained cells were analyzed by fluorescenceactivated cell sorting (FACS) on a FACScan flow cytometer (Becton Dickinson and Co., Franklin Lakes, NY, USA) for the relative DNA content based on fluorescence.

Asp-Glu-Val-Asp-ase (DEVDase) activity assays. The cells were washed twice with PBS and incubated in lysis buffer. Insoluble materials were removed by centrifugation $(15,115 \mathrm{x} \mathrm{g}$ for $10 \mathrm{~min}$ at $4^{\circ} \mathrm{C}$ ), and protein concentrations were quantified using the Bio-Rad protein assay (Bio-Rad, Hercules, CA, USA). Caspase activities were determined with colorimetric assays using caspase-3 (DEVDase) and caspase-8 activity assay kits (Calbiochem, San Diego, CA, USA), according to the manufacturer's instructions. DEVDase assays were performed in 96-well microtiter plates by incubating $20 \mu \mathrm{g}$ of cell lysates in $100 \mu \mathrm{l}$ of reaction buffer (1\% NP-40, $20 \mathrm{~mm}$ Tris- $\mathrm{HCl}, \mathrm{pH} 7.5,137 \mathrm{~mm}$ $\mathrm{NaCl}, 10 \%$ glycerol) containing each caspase substrate $(5 \mu \mathrm{m})$. The lysates were incubated at $37^{\circ} \mathrm{C}$ for $2 \mathrm{~h}$. Thereafter, absorbance at $405 \mathrm{~nm}$ was measured using a spectrophotometer.

RNA isolation and reverse transcriptase-polymerase chain reaction ( $R T-P C R)$. Total cellular RNA was extracted using TRIzol reagent (Life Technologies Corp., Carlsbad, CA, USA). Single-strand cDNA was synthesized from $2 \mu \mathrm{g}$ of total RNA using M-MLV reverse transcriptase (Promega, Madison, WI, USA) according to the manufacturer's instrucions. The cDNA for $\mathrm{CFLIP}$ and $\beta$-actin was amplified using the following specific primers: cFLIP (sense) 5'-CCCAGTGGAC AGCGAGC-3' and (antisense) 5'-ACTGCAGGCTTCCTGTGCGC-3', and actin (sense) 5'-GGCATCGTCACCAACTGGGAC-3' and (antisense) 5'-CGATTTCCCGCTCGGCCGTGG-3'. PCR amplification was carried out as follows: 1 cycle $\left(94^{\circ} \mathrm{C}, 3 \mathrm{~min}\right) ; 30$ cycles $\left(94^{\circ} \mathrm{C}, 45 \mathrm{sec} ; 59^{\circ} \mathrm{C}, 45 \mathrm{sec}\right.$; and $\left.72^{\circ} \mathrm{C}, 1 \mathrm{~min}\right)$; and 1 cycle $\left(72^{\circ} \mathrm{C}\right.$, $10 \mathrm{~min})$. PCR products were analyzed by agarose gel electrophoresis and visualized by ethidium bromide.

\section{Results}

FPDHP induces apoptosis and DNA fragmentation in Caki cells, and attenuates the growth of various cancer cell lines. Initially, we investigated the cell death-inducing ability of FPDHP in Caki cells. The Caki cells were treated for $24 \mathrm{~h}$ with the indicated concentrations of FPDHP and then evaluated for morphological changes and DNA content following propidium iodide staining. As shown in Fig. 1B, compared with the control cells, $24 \mathrm{~h}$ of treatment with FPDHP $(8 \mu \mathrm{M})$ induced cell detachment and morphological changes contributing to apoptosis and increased the cell populations in the sub-G1 phase. Treatment with FPDHP $(8 \mu \mathrm{M})$ markedly increased the amount of DNA fragmentation (Fig. 1C). In order to evaluate the anticancer effects of FPDHP, various types of cancer cells, such as Caki, HT29, MDA-MB-231 and 
A

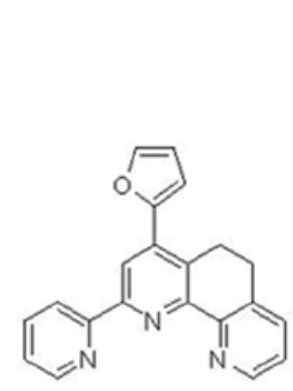

B

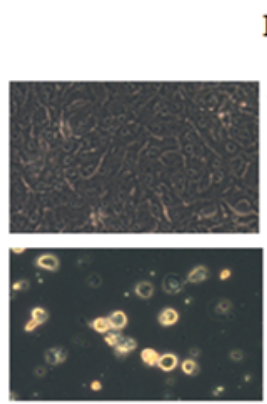

FPDHP Sub-Gl

$(\mu \mathrm{M})$

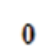

0

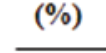

2.1

8

35.1

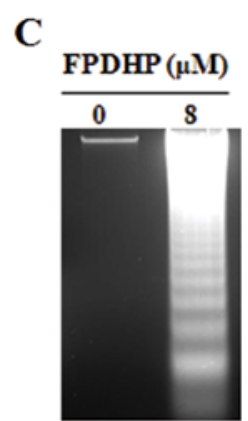

D

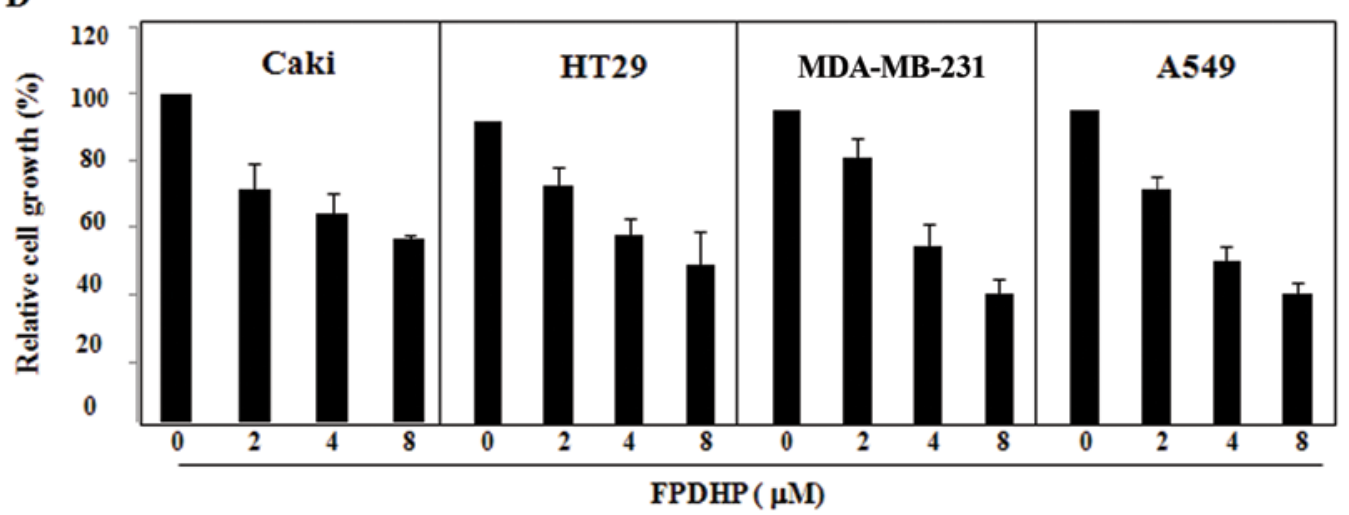

Figure 1. The structure and anticancer properties of 4-(furan-2-yl)-2-(pyridin-2-yl)-5,6-dihydro-1,10-phenanthroline (FPDHP). (A) Chemical structure of FPDHP. (B) Cell morphology and flow cytometric analysis of apoptotic cells. Caki cells were treated for $24 \mathrm{~h}$ with or without FPDHP $(8 \mu \mathrm{M})$ and then evaluated for DNA content following propidium iodide staining. (C) DNA fragmentation analysis. DNA was extracted from Caki cells treated for $24 \mathrm{~h}$ with or without FPDHP $(8 \mu \mathrm{M})$ and analyzed on a 2\% agarose gel. (D) Effects of FPDHP on the growth of Caki, HT29, MDA-MB-231 and A549 cells. Cancer cells were treated with or without FPDHP at the indicated concentrations for $24 \mathrm{~h}$, and cell growth was measured by XTT assay. Data are the mean values obtained from 3 independent experiments and bars represent standard deviation.

A549 cells were treated with FPDHP for $24 \mathrm{~h}$, followed by the measurement of the respective cell growth by XTT assay. FPDHP attenuated the growth of all the cells tested in a dosedependent manner (Fig. 1D).

Apoptosis induced by FPDHP is dependent on caspase activation. Considering that caspases play key roles in apoptosis, we then examined whether FPDHP triggers the activation of caspases in Caki cells. FPDHP increased the DEVDase activity in Caki cells, indicating the activation of caspases by this small molecule (Fig. 2A). We then evaluated caspase dependency in FPDHP-mediated Caki cell death. For this purpose, we treated the Caki cells with or without FPDHP in the presence or absence of $\mathrm{z}$-VAD for $24 \mathrm{~h}$, followed by the measurement of the cell populations in the sub-G1 phase and the cellular levels of pro-caspase-3, PLC- $\gamma 1$ and ERK in the conditioned cells by FACS and western blot analysis. FPDHP increased the population of Caki cells in the sub-G1 phase, which was largely suppressed by pre-treatment with z-VAD, a pan-caspase inhibitor (Fig. 2B). FPDHP decreased the cellular levels of pro-caspase-3 (inactive), while it increased the cleaved forms of caspase- 3 (active) and PLC- $\gamma 1$, a downstream substrate of caspases, in the Caki cells (Fig. 2C). However, the FPDHP-mediated decrease in the levels of pro-caspase- 3 and the increase in the levels of caspase- 3 and PLC- $\gamma 1$ were not observed in the cells pre-treated with $\mathrm{z}$-VAD. These results suggest that the activation of caspase- 3 is a key executioner of the apoptosis induced by FPDHP.
FPDHP downregulates cFLIP and p-Akt expression in Caki cells, and overexpression of cFLIP partially inhibits FPDHP-mediated apoptosis. To further evaluate the apoptosisinducing mechanisms of FPDHP, we then determined whether FPDHP affects the expression levels of cell growth- and/or apoptosis-related signaling proteins in the Caki cells. As shown in Fig. 3A, compared with the control (untreated cells), FPDHP decreased the cellular levels of cFLIP, p-Akt and total Akt. FPDHP, however, did not alter the expression levels of Bcl-2. Distinctly, pre-treatment with z-VAD blocked the downregulation of p-Akt, but not that of cFLIP, which was induced by FPDHP. Furthermore, FPDHP also suppressed the mRNA expression of cFLIP (Fig. 3B). These results suggest that treatment with FPDHP leads to the downregulation of cFLIP expression at the transcriptional levels. To determine whether the downregulation of cFLIP and p-Akt contributes to FPDHPmediated apoptosis, we treated the control cells (Caki/vector), cFLIP overexpressing cells (Caki/cFLIP) or the Akt overexpressing cells (Caki/Akt) with or without FPDHP, followed by the measurement of cFLIP or p-Akt expression levels and the populations of cells in the sub G1 phase by western blot analysis and flow cytometry, respectively. The overexpression of cFLIP, but not that of p-Akt, led to a partial attenuation of FPDHP-mediated apoptosis (Fig. 3C and D).

FPDHP rapidly induces cell detachment and increases the number of apoptotic cells in the detached cells. Considering that FPDHP led to the detachment of Caki cells from the 
A

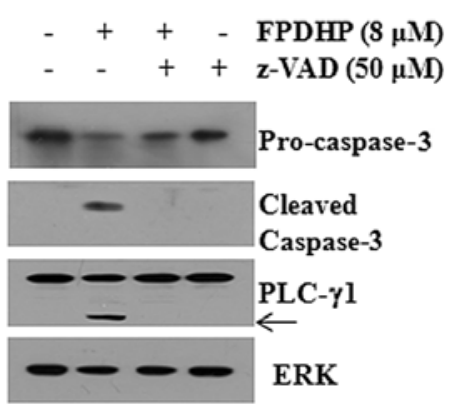

B

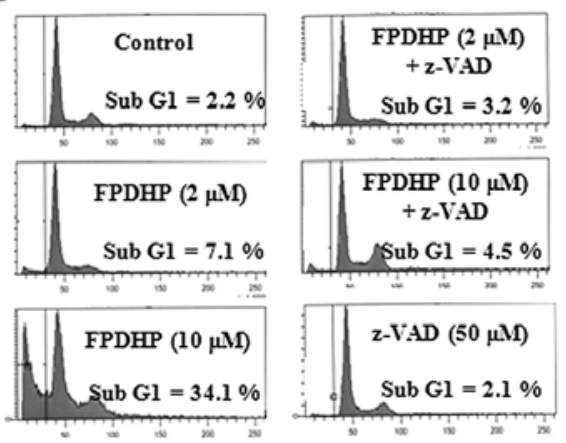

C

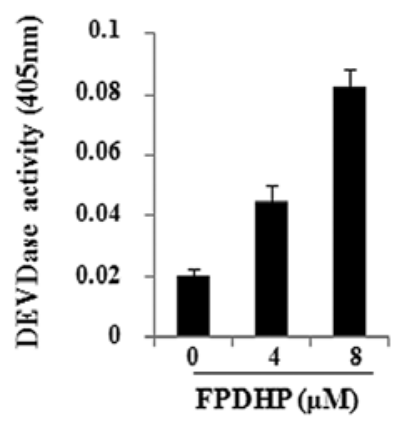

Figure 2. Effect of 4-(furan-2-yl)-2-(pyridin-2-yl)-5,6-dihydro-1,10-phenanthroline (FPDHP) and/or z-VAD (a pan-caspase inhibitor) on apoptosis of Caki cells. (A) Caki cells were treated with or without FPDHP $(8 \mu \mathrm{M})$ in the presence or absence of $\mathrm{z}$-VAD for $24 \mathrm{~h}$. Whole cell lysates were isolated and analyzed by western blot analysis with specific antibody. The cleaved form of phospholipase C (PLC)- $\gamma 1$ is indicated by an arrow. Extracellular signal-related kinase (ERK) was used as a protein loading control. Results are representative of 3 independent experiments. (B) Caki cells were treated with or without FPDHP (8 $\mu$ M) in the presence or absence of z-VAD for $24 \mathrm{~h}$. Whole cell lysates were isolated and analyzed by DEVDase activity. Data are presented as the mean values from 3 independent experiments, and bars represent standard deviation. (C) Caki cells were treated with or without FPDHP ( $8 \mu \mathrm{M})$ in the presence or absence of z-VAD for $24 \mathrm{~h}$, followed by the measurement of the cell populations in the sub-G1 phase by flow cytometric analysis. Results are representative of 3 independent experiments.

A

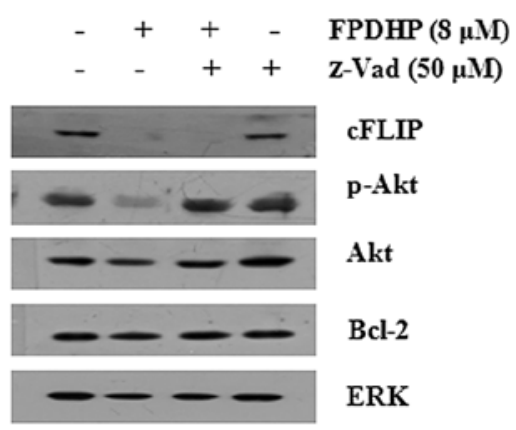

B

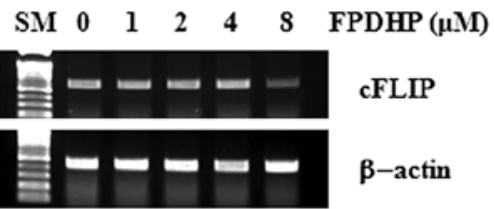

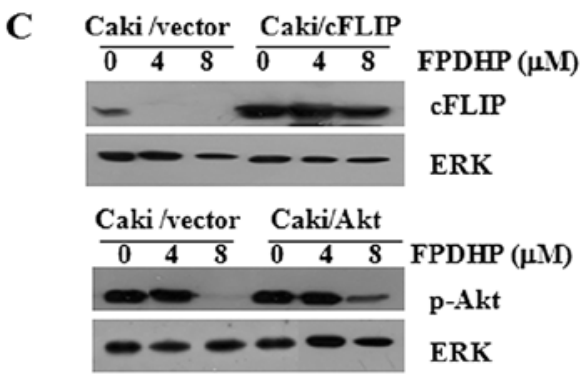

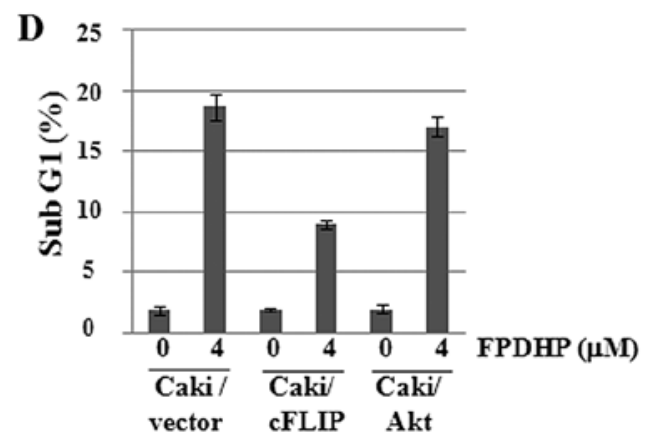

Figure 3. Apoptosis induced by 4-(furan-2-yl)-2-(pyridin-2-yl)-5,6-dihydro-1,10-phenanthroline (FPDHP) is partially associated with the decrease in cellular FLICE-like inhibitory protein (cFLIP) expression in Caki cells. (A) Caki cells were treated with or without FPDHP (8 $\mu \mathrm{M})$ in the presence or absence of $\mathrm{z}-\mathrm{VAD}$ (a pan-caspase inhibitor) for $24 \mathrm{~h}$. Whole cell lysates were isolated and analyzed by western blot analysis with specific antibody. Results are representative of 3 independent experiments. (B) Caki cells were treated with or without FPDHP $(8 \mu \mathrm{M})$ in the presence or absence of z-VAD for 6 h. Total RNA was extracted and analyzed by RT-PCR with specific primers to detect the mRNA levels of cFLIP and $\beta$-actin. Results are representative of 3 independent experiments. (C) Caki/ vector and Caki/cFLIP cells were treated with or without FPDHP $(4$ or $8 \mu \mathrm{M})$ for $24 \mathrm{~h}$. Whole cell lysates were isolated and analyzed by western blot analysis with specific antibody. Results are representative of 3 independent experiments. (D) Caki/vector and Caki/cFLIP cells were treated with or without FPDHP (4 $\mu \mathrm{M})$ for $24 \mathrm{~h}$, followed by the measurement of the cell populations in the sub-G1 phase by flow cytometric analysis. Results are representative of 3 independent experiments.

cell culture dish, particularly during the early treatment periods (Fig. 1B), we then analyzed the cell detachmentinducing capacity and kinetics of FPDHP and its association with FPDHP-mediated apoptosis. To this end, we treated the Caki cells with or without FPDHP for the designated periods of time, and harvested and counted the numbers of attached cells (ACs) and detached cells (DCs) separately at each time point. The numbers of DCs were increased in a time-dependent manner, and most of the cells were detached within $8 \mathrm{~h}$ after FPDHP treatment (Fig. 4A). To evaluate whether cell detach- ment affects apoptosis, the ACs and DCs were harvested separately from the Caki cells treated with FPDHP $(8 \mu \mathrm{M})$ for $4 \mathrm{~h}$, and the populations of cells in the Sub-G1 phase in the ACs and DCs were measured by flow cytometry. The Sub-G1 ratio of the DCs was higher than that of the ACs (Fig. 4B). These results suggest that cell detachment induced by FPDHP may affect the induction of apoptosis. To determine whether caspase activated by FPDHP affects FPDHP-mediated cell detachment and apoptosis, the Caki cells were treated with or without FPDHP in the presence or absence of z-VAD for 

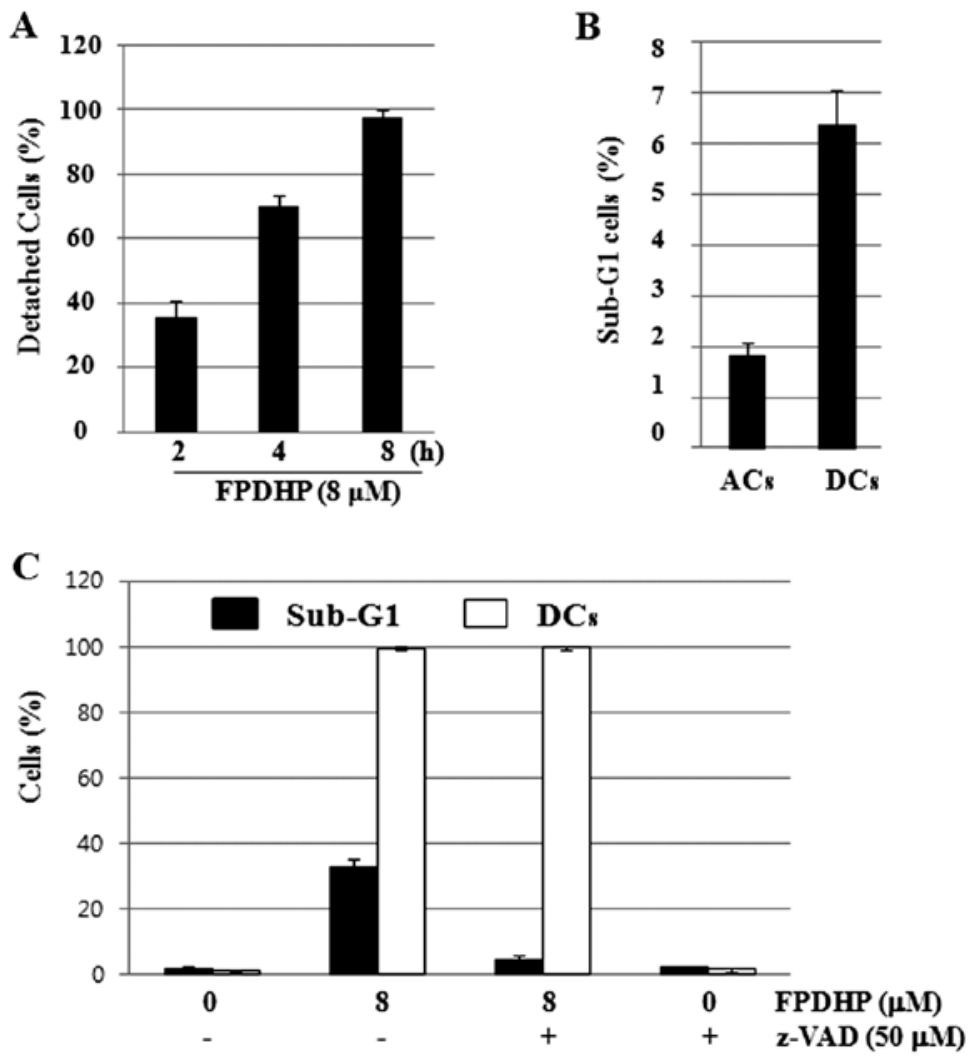

Figure 4. Effect of 4-(furan-2-yl)-2-(pyridin-2-yl)-5,6-dihydro-1,10-phenanthroline (FPDHP) on cell detachment-mediated apoptosis in Caki cells. (A) Caki cells were treated with or without FPDHP $(8 \mu \mathrm{M})$ for 2,4 and $8 \mathrm{~h}$. Detached cells (DCs) in the supernatant and adherent cells (ACs) in the cell culture dish, were harvested at the indicated time points, and the ratio of DCs (DCs/total cells x100) was calculated. (B) Caki cells were treated with or without FPDHP ( $4 \mu \mathrm{M})$ for $4 \mathrm{~h}$. ACs and DCs were harvested separately, followed by the measurement of the cell populations in the sub-G1 phase by flow cytometric analysis. (C) Caki cells were treated with or without FPDHP $(8 \mu \mathrm{M})$ in the presence or absence of z-VAD (a pan-caspase inhibitor) for $24 \mathrm{~h}$. DCs were harvested and analyzed by cell counting or flow cytometric analysis.

$24 \mathrm{~h}$ and the numbers of DCs and the populations of cells in the Sub-G1 phase were measured by cell counting and flow cytometry, respectively. As shown in Figs. 1B and 4A, respectively, the numbers of DCs increased in a time-dependent manner, and most of the cells were detached within $8 \mathrm{~h}$ after FPDHP treatment, regardless of z-VAD pre-treatment (data not shown). Twenty-four hours later, the cells treated with both FPDHP and z-VAD were almost detached, but apoptosis was markedly suppressed by z-VAD (Fig. 4D).

\section{Discussion}

Increasing the understanding of the underlying molecular events regulating several different cell death mechanisms, such as apoptosis, necroptosis and autophagic cell death has opened many new possibilities in the development of novel anticancer agents (13-15). Among several death mechanisms, the induction of apoptosis is the most important method in the treatment of cancers, as cancer is one of the scenarios where too little apoptosis occurs, resulting in cancer cells that will not die, and defects at any point along the apoptosis pathways lead to the malignant transformation of the affected cells, tumor metastasis and resistance to anticancer drugs $(16,17)$. Therefore, a number of apoptosis-modulating drugs have been developed (18). Previously, we designed and synthesized FPDHP as a phenanthroline derivative, and demonstrated its topoisomerase I inhibitory activity and cytotoxicity against several human cancer cell lines (12). In this study, to the best of our knowledge, we report for the first time the anti-growth and pro-apoptotic effects of FPDHP on Caki human renal cancer cells through multiple mechanisms, including caspase-dependent apoptosis and the downregulation of cFLIP and the caspase-independent cell detachment, which may suggest that FPDHP is a novel inducer of apoptosis.

The induction of apoptosis is associated with a variety of proteins and/or factors. Among these, caspase- 3 is one of the most important cell death-inducing proteases that cleave a number of proteins essential for cell survival $(19,20)$. In this study, we demonstrate that FPDHP induces Caki cell death and attenuates the growth of various cancer cell lines. In particular, the present study clearly demonstrates that FPDHP stimulates the activity of caspase- 3 , the cleavage of PLC- $\gamma 1$, and increases the numbers of Caki cells in the sub-G1 phase. Importantly, we demonstrate that pre-treatment with the pancaspase inhibitor, z-VAD, significantly inhibits the majority of the anticancer responses induced by FPDHP, suggesting that caspases play critical roles in FPDHP-mediated apoptosis in Caki cells. FPDHP also induced cell death in other cancer cells, such as HT29 and A549 cells, and these cell deaths were also inhibited by pre-treatment with z-VAD (data not shown). These results suggest that FPDHP has an ability to induce caspase-dependent apoptosis in numerous cancer cells.

To further delineate the regulatory mechanisms underlying the killing effect on Caki cells by FPDHP, we measured the expression levels of proteins associated with cell growth and/ 
or apoptosis in the Caki cells. In this study, we demonstrate that FPDHP decreases the expression of cFLIP, known as cellular FLICE-inhibitory protein, by transcriptional repression in Caki cells. However, in this study, pharmacological inhibition experiments revealed that pre-treatment with z-VAD did not attenuate the downregulation of cFLIP in the Caki cells treated with FPDHP. These results thus indicate that the cFLIP downregulation is caspase-independent. Furthermore, the overexpression of cFLIP attenuated apoptosis induced by FPDHP. cFLIP has been identified as an inhibitor of apoptosis triggered by the engagement of death receptors, such as Fas or TRAIL, and abnormal cFLIP expression has been identified in various types of cancer $(21,22)$. Therefore, this suggests that the transcriptional downregulation of cFLIP by FPDHP may be important for the induction of FPDHP-mediated apoptosis.

During the observation of cellular morphological changes under a microscope, we have found that FPDHP significantly induced cell detachment from the early incubation time points after FPDHP treatment. Cell detachment-induced cell death is known as anoikis. A number of studies have shown that when cancer cells are detached from the original cancer mass, the induction of anoikis is important for the prevention of cancer metastasis $(23,24)$. In this study, we demonstrated that FPDHP induced the detachment of the majority of Caki cells within $8 \mathrm{~h}$ of incubation, and the sub-G1 ratio of the DCs was much higher than that of the ACs (Fig. 4A). Moreover, we further demonstrated that pre-treatment with z-VAD inhibited apoptosis, but not the cell detachment induced by FPDHP. These results thus suggest that FPDHP downregulates certain types of cell adhesion molecules in a caspase-independent manner, and that cell detachment induced by FPDHP is associated with the induction of caspase-dependent apoptosis.

Taken together, the results from the present study demonstrate that FPDHP induces apoptosis in Caki cells through the activation of caspases, the caspase-dependent downregulation of cFLIP and cell detachment. These novel properties of FPDHP which functions as a topoisomerase inhibitor suggest that this compound is worthy of being developed as a novel anticancer agent.

\section{References}

1. Pommier Y: DNA topoisomerase I inhibitors: chemistry, biology, and interfacial inhibition. Chem Rev 109: 2894-2902, 2009.

2. Wang JC: DNA topoisomerases. Annu Rev Biochem 65: 635-692, 1996.

3. Nitiss JL: Investigating the biological functions of DNA topoisomerases in eukaryotic cells. Biochim Biophys Acta 1400: 63-81, 1998.

4. Kellner U, Rudolph P, Parwaresch R, et al: Human DNA-topoisomerases - diagnostic and therapeuticimplications for cancer. Onkologie 23: 424-430, 2000.
5. Singh SK, Ruchelman AL, Li TK, et al: Nitro and amino substitution in the D-ring of 5-(2-dimethylaminoethyl)- 2,3-methylenedioxy-5H-dibenzo[c,h)[1,6)naphthyridin-6-ones: effect on topoisomerase-I targeting activity and cytotoxicity. J Med Chem 46: 2254-2257, 2003

6. Forterre P, Gribaldo S, Gadelle D and Serre MC: Origin and evolution of DNA topoisomerases. Biochimie 89: 427-446, 2007.

7. Bailly C: Contemporary challenges in the design of topoisomerase II inhibitors for cancer chemotherapy. Chem Rev 112: 3611-3640, 2012.

8. Jeong BS, Choi H, Kwak YS and Lee ES: Synthesis of 2,4,6-tripyridyl pyridines, and evaluation of their antitumor cytotoxicity, topoisomerase I and II inhibitory activity, and structure-activity relationship. Bull Korean Chem Soc 32: 3566-3570, 2011

9. Thapa U, Thapa P, Karki R, et al: Synthesis of 2,4-diaryl chromenopyridines and evaluation of their topoisomerase I and II inhibitory activity, cytotoxicity, and structure-activity relationship. Eur J Med Chem 46: 3201-3209, 2011.

10. Thapa P, Karki R, Yoo HY, et al: 2,4-Diaryl-5,6-dihydro1,10-phenanthroline and 2,4-diaryl-5,6-dihydrothieno[2,3-h) quinoline derivatives for topoisomerase I and II inhibitory activity, cytotoxicity, and structure-activity relationship study. Bioorg Chem 40: 67-78, 2012.

11. Lee SH, Van HT, Yang SH, et al: Molecular design, synthesis and docking study of benz[b)oxepines and 12-oxobenzo[c)phenanthridinones as topoisomerase 1 inhibitors. Bioorg Med Chem Lett 19: 2444-2447, 2009.

12. Thapa $P$ and Lee ES: 2,4-Diaryl-5,6-dihydro-1,10-phenanthrolines with furyl or thienyl moiety at 4-position: synthesis, topoisomerase I and II inhibitory activity, and cytotoxicity. Bull Korean Chem Soc 33: 1769-1772, 2012.

13. Long JS and Ryan KM: New frontiers in promoting tumour cell death: targeting apoptosis, necroptosis and autophagy. Oncogene 31: 5045-5060, 2012

14. Yu X, Deng Q, Bode AM, et al: The role of necroptosis, an alternative form of cell death, in cancer therapy. Expert Rev Anticancer Ther 13: 883-893, 2013

15. Reyjal J, Cormier K and Turcotte S: Autophagy and cell death to target cancer cells: exploiting synthetic lethality as cancer therapies. Adv Exp Med Biol 772: 167-188, 2014.

16. Mashima T and Tsuruo T: Defects of the apoptotic pathway as therapeutic target against cancer. Drug Resist Updat 8: 339-343, 2005.

17. Rodriguez-Nieto $S$ and Zhivotovsky B: Role of alterations in the apoptotic machinery in sensitivity of cancer cells to treatment. Curr Pharm Des 12: 4411-4425, 2006.

18. Ocker $\mathrm{M}$ and Höpfner M: Apoptosis-modulating drugs for improved cancer therapy. Eur Surg Res 48: 111-120, 2012.

19. Emoto Y, Manome Y, Meinhardt G, et al: Proteolytic activation of protein kinase $\mathrm{C}$ delta by an ICE-like protease in apoptotic cells. EMBO J 14: 6148-6156, 1995.

20. Porter AG and Jänicke RU: Emerging roles of caspase-3 in apoptosis. Cell Death Differ 6: 99-104, 1999.

21. Micheau O: Cellular FLICE-inhibitory protein: an attractive therapeutic target? Expert Opin Ther Targets 7: 559-573, 2003.

22. Li X, Pan X, Zhang H, et al: Overexpression of cFLIP in head and neck squamous cell carcinoma and its clinicopathologic correlations. J Cancer Res Clin Oncol 134: 609-615, 2008.

23. Chiarugi P and Giannoni E: Anoikis: a necessary death program for anchorage-dependent cells. Biochem Pharmacol 76: 1352-1364, 2008.

24. Simpson CD, Anyiwe K and Schimmer AD. Anoikis resistance and tumor metastasis. Cancer Lett 272: 177-185, 2008. 